\title{
Understanding the size growth of massive galaxies through stellar populations
}

\section{Ferreras ${ }^{1}$, I. Trujillo ${ }^{2}$, E. Mármol-Queraltó ${ }^{3}$, P. Pérez-González ${ }^{4}$ and the SHARDS team}

\author{
${ }^{1}$ Mullard Space Science Laboratory, University College London, UK \\ ${ }^{2}$ Instituto de Astrofísica de Canarias \& Universidad de La Laguna, Spain \\ ${ }^{3}$ Institute for Astronomy, Royal Observatory, Edinburgh, UK \\ ${ }^{4}$ Departamento de Astrofísica, Universidad Complutense de Madrid, Spain \\ email: i.ferreras@ucl.ac.uk
}

\begin{abstract}
Massive early-type galaxies undergo a significant process of evolution with redshift on the stellar mass vs size plane. Furthermore, this trend does not depend on the age of their stellar populations. Therefore, such an evolution should involve processes that do not include a significant amount of star formation, leaving (mostly) dry mergers as the main growth channel. By studying close pairs involving a massive galaxy, one can quantify the role of mergers on the growth of massive galaxies. A recent study based on the SHARDS dataset reveals that minor mergers cannot be the dominant mechanism to explain the bulk of size growth in these systems. Merging is found to provide a constant fractional growth rate of $\sim 10 \%$ per Gyr from redshift $\mathrm{z}=1$, corresponding to an overall stellar mass increase of $2 \times$ between $\mathrm{z}=1$ and $\mathrm{z}=0$.
\end{abstract}

Keywords. galaxies:evolution, galaxies:formation

\section{Introduction}

The formation history of massive early-type galaxies has posed one of the most stringent constraints to models of galaxy formation. Their quiescent stellar populations and chemical composition reveal an intense, short-lived and early process for the formation of the cores of these galaxies (see, e.g. de la Rosa et al. 2011). Such a result contrasts with the mass assembly history, which - in a bottom-up hierarchical context - require a more extended formation process. Moreover, this type of galaxies have been found to evolve on the mass-size plane from $\mathrm{z} \sim 2$ until present time. Galaxies with the same stellar mass are more compact at higher redshift (see, e.g. Daddi et al. 2005; Trujillo et al. 2006; van Dokkum et al. 2010). Several mechanisms have been proposed to explain this trend, most notably through the expulsion of gas (Fan et al. 2008), via progenitor bias (van der Wel et al. 2008) or from merging (Khochfar \& Silk 2009).

Fig. 1 (adopted from Trujillo et al. 2011) compares the average stellar ages of earlytype galaxies at moderate redshift $(\mathrm{z} \lesssim 1)$ from the HST/PEARS survey (Ferreras et al. 2009 ) with a local sample of early-type galaxies from SDSS, extracted from the catalogue of Nair \& Abraham (2010). In addition, we use the expected age distribution, constrained from spectral fitting, to derive their ages at the redshifts shown in the different panels of the figure. The local (SDSS) sample is shown as coloured triangles, split (red/blue) with respect to age (old/young) at the median age of the sample, in each redshift bin. The evident lack of an age segregation on the mass-size plane is at odds with the gas and progenitor bias scenarios, as those options would introduce a significant trend between the size of an early-type galaxy, at fixed mass, and its stellar age.

The remaining growth channel compatible with the observations - (mostly dry) merging - can be explored through merger histories. Instead of "backtracking" the formation 


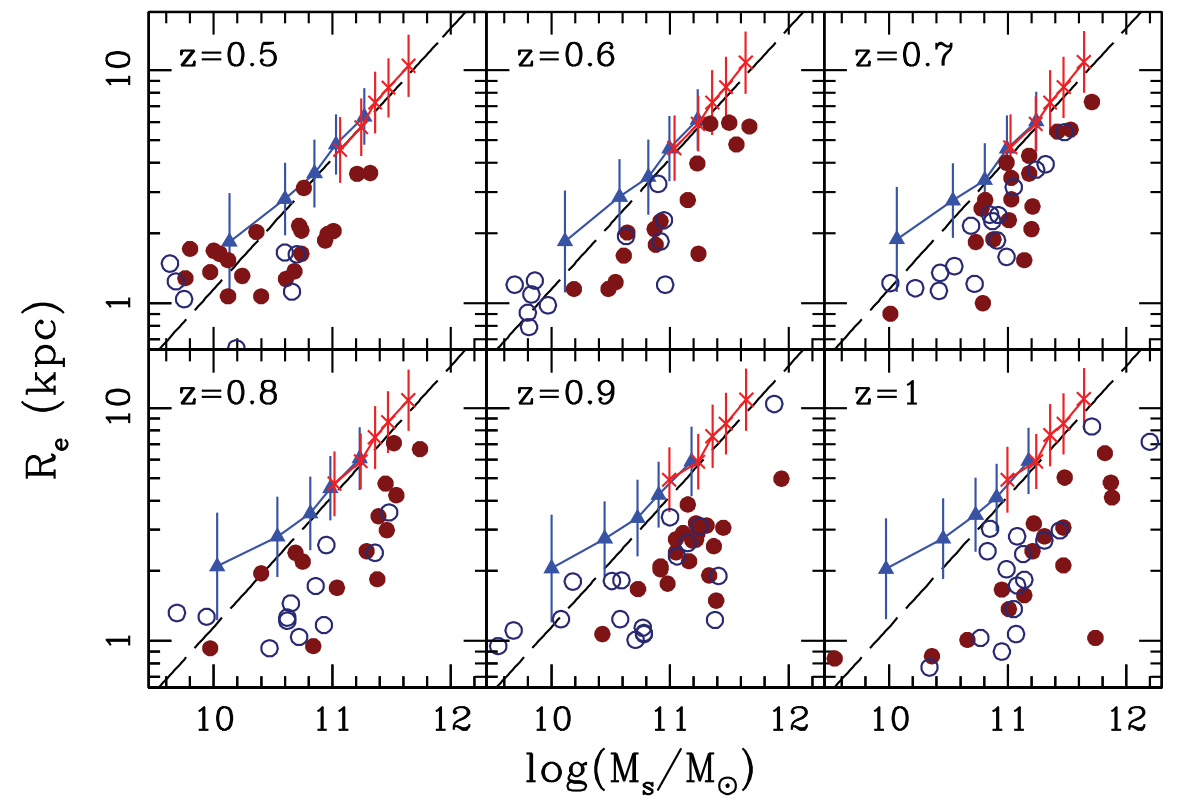

Figure 1. Distribution on the mass-size plane of early-type galaxies at intermediate redshift with respect to stellar age. The trend at zero redshift (from SDSS) is shown with triangles, separated into young (blue) and old (red) subsamples. The red solid (blue open) dots correspond to the HST/PEARS sample (Ferreras et al. 2009), split with respect to stellar age into old (young) galaxies. No significant difference is evident. Adapted from Trujillo et al. (2011).

history with observational data of galaxies at low redshift, it is possible to forward-model the growth process by the analysis of merging progenitors. Close pairs of galaxies can be used for this task (see, e.g. Patton et al. 2000). In this contributed talk, we present the main results from a recent analysis of close pairs involving massive galaxies at moderate redshift (Ferreras et al. 2014).

\section{Sample}

The sample is extracted from the Survey for High-z Absorption Red and Dead Sources (SHARDS), a dataset consisting of deep $(<26.5 \mathrm{AB})$ photometry through 24 medium band $\left(\Delta \lambda \sim 150 \AA\right.$ ) filters over a $130 \operatorname{arcmin}^{2}$ region towards the HDFN (Pérez-González et al. 2013). Medium band photometric data enables us to achieve accurate photometric redshifts $(\Delta z /(1+z) \sim 0.055)$ without the completeness issues present in spectroscopic surveys. Moreover, we can use the data effectively as low-resolution $(\mathrm{R} \sim 50)$ photo-spectra in order to explore the properties of the stellar populations. From this sample, we select a set of massive galaxies over the redshift interval $0.3<\mathrm{z}<1.3$, and target those systems with companions at the same redshift - within the uncertainties - and inside a projected physical radius of $100 h_{70}^{-1} \mathrm{kpc}$. Such systems can be considered potential mergers, contributing to the growth of massive galaxies. The depth of the survey allows us to define a complete sample of close pairs down to a mass ratio 1:20 as a conservative estimate, and 1:100 in the expected scenario where lower mass galaxies have younger stellar populations (and hence lower M/L ratios). The interested reader is referred to Ferreras et al. (2014) for further details about the sample selection. 


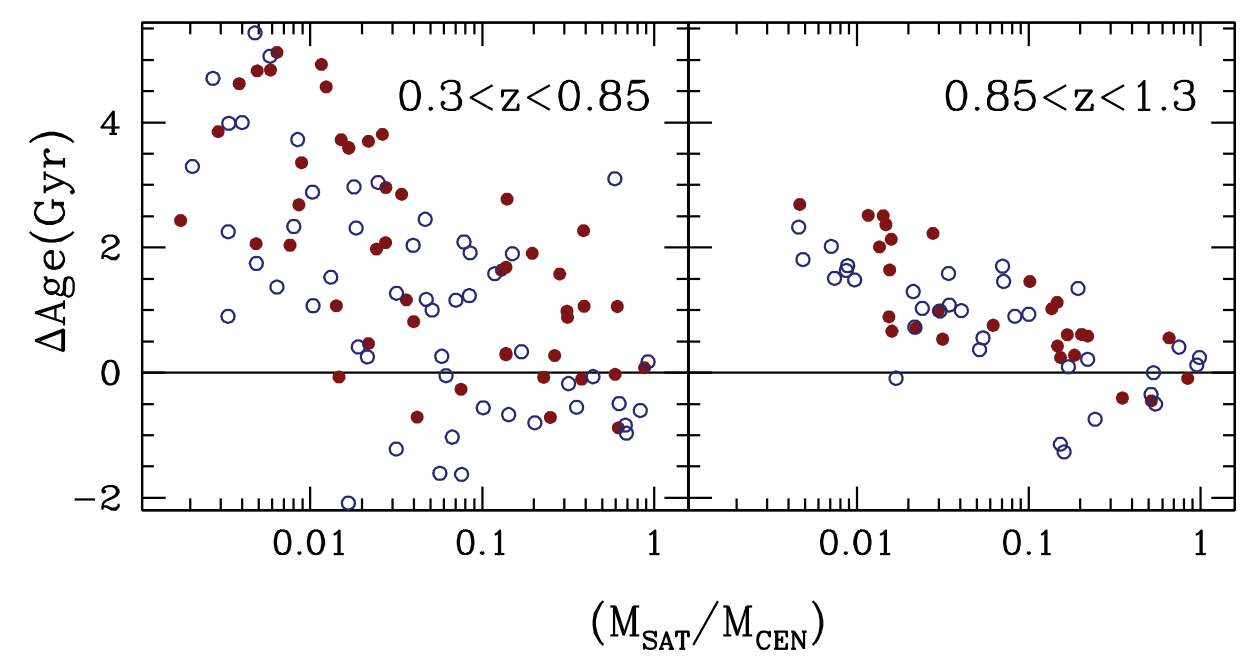

Figure 2. Age difference between central and satellite galaxy in close pairs involving at least one massive system (stellar mass $\geqslant 10^{11} M_{\odot}$ ). The result is shown with respect to the mass ratio, split into two redshift bins, as labelled. Red solid (open blue) dots correspond to E/S0 (disc/irregular) galaxies. Adapted from Ferreras et al. (2014).

\section{Results}

A grid of composite population synthesis models is applied to the photo-spectra of the sample to determine the average stellar ages of the centrals and their close companions. Such an exercise throws light on the expected radial distribution of stellar ages in massive galaxies at low redshift. Fig. 2 shows the stellar age difference between the central and the satellites, as a function of the mass ratio. The sample is split with respect to the morphological appearance of the centrals, as spheroidal (red solid dots) or disc/irregular (blue open circles). The figure confirms that these galaxies do not have any peculiarity with respect to the general sample, i.e. the lower mass galaxies are younger, according to the general mass-age trend. Note the difference between the spheroidals and the late-type systems: as the latter have younger populations, their central-satellite age difference is smaller with respect to those with an early-type central. Given that the stellar component from a minor merger will mostly populate the outer regions of a massive galaxy, a large number of minor mergers would lead to a significantly younger population in the outskirts of massive galaxies at $\mathrm{z} \sim 0$, a scenario ruled out by the observations (see, e.g. la Barbera et al. 2012).

In order to quantify in more detail the contribution from major or minor mergers, we determine from our sample the stellar mass growth as a function of the mass ratio between central and satellite. Fig. 3 presents our results, given as the fractional mass growth rate, showing the complete sample (red crosses) and the subsample consisting of major mergers (i.e. mass ratio above 0.3). We also show in the figure the low-redshift estimates derived from the SDSS dataset by Ruiz et al. (2014). Note that the fractional growth rate via merging stays approximately constant, at $\Delta M / M \sim 10 \%$ per Gyr. This result stems from the weak/no evolution trend with redshift of the fraction of massive galaxies with close pairs (see also Mármol-Queraltó 2012, 2013). For reference, the vertical axis on the right hand side of Fig. 3 shows the equivalent stellar mass growth between $z=1$ and $z=0$. We obtain a growth rate $\sim 2 \times$. Remarkably, the contribution from 1:3 mergers is very large, implying that minor mergers cannot represent the dominant growth channel in massive galaxies. 


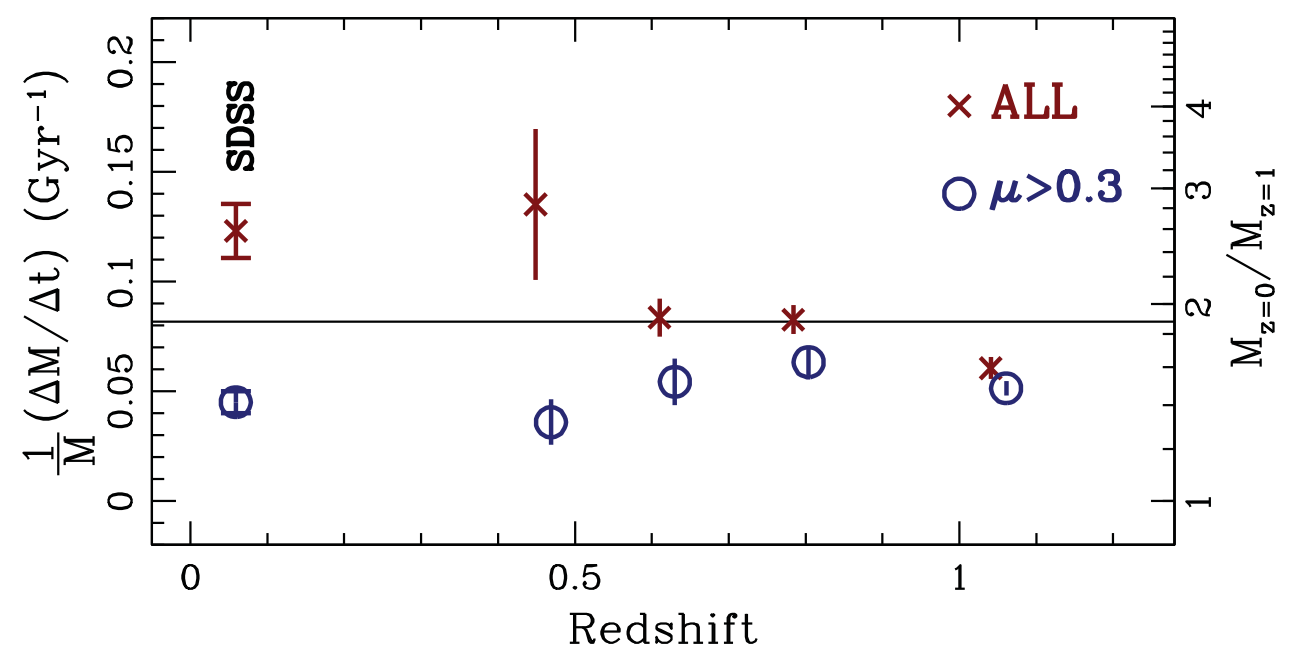

Figure 3. Redshift evolution of the mass growth of massive galaxies expected from mergers, using close pairs as a proxy of the merging history. The results are shown both for the full sample (robustly complete down to a mass ratio of 1:20, and including satellites down to 1:100, red crosses) and for major merger progenitors (mass ratio above 1:3, blue open circles). This analysis includes the effect of dynamical friction according to the prescriptions suggested by Jiang et al. (2014). Note the preponderance of the major merging component ratios in the expected growth. The data points at $\mathrm{z}=0$ (labelled SDSS) correspond to the analysis of Ruiz et al. (2014). Adapted from Ferreras et al. 2014.

\section{References}

Bruzual, G. \& Charlot, S. 2003, MNRAS, 344, 1000

Daddi, E, et al. 2005, ApJ, 626, 680

de la Rosa, I. G., et al. 2011, MNRAS, 418, L74

Fan, L., et al. 2008, ApJ, 689, L101

Ferreras, I., et al. 2009, ApJ, 706, 158

Ferreras, I., et al. 2014, MNRAS, 444, 906

Jiang C. Y., Jing Y. P., Han J. 2014, ApJ, 790, 7

Khochfar, S. \& Silk, J. 2009, MNRAS, 397, 506

La Barbera, F., et al. 2012, MNRAS, 426, 2300

Mármol-Queraltó, E., et al. 2012, MNRAS, 422, 2187

Mármol-Queraltó, E., et al. 2013, MNRAS, 429, 792

Nair, P. B. \& Abraham, R. G. 2010, ApJS, 186, 427

Patton, D. R., et al. 2000, ApJ, 536, 153

Pérez-González, P. G., et al. 2013, ApJ, 762, 46

Ruiz, P., et al. 2014, MNRAS, 442, 347

Trujillo, I., et al. 2006, ApJ, 650, 18

Trujillo, I., Ferreras, I., \& de la Rosa, I. G. 2011, $M N R A S, 415,3903$

van Dokkum, P. G., et al. 2010, ApJ, 709, 1018

van der Wel, A., et al. 2008, ApJ, 688, 48 